\title{
Isolated Management of the Aging Neck
}

\author{
Juan D. Mejia, M.D., ${ }^{1}$ Farzad R. Nahai, M.D., ${ }^{2}$ Foad Nahai, M.D., F.A.C.S., ${ }^{2}$ \\ and Adeyiza O. Momoh, M.D. ${ }^{3}$
}

\section{ABSTRACT}

The contour of the neck is a very important determinant of facial aesthetics. Precise knowledge of neck anatomy is essential for adequate planning and execution of this procedure. There are three anatomic and surgical planes involved in the management of the aging neck; the superficial plane (subcutaneous fat), the intermediate plane (platysma muscles and the fat between the two muscles), and the deep plane (subplatysmal fat, the anterior belly of the digastric muscles, and the submandibular glands). These planes need to be thoroughly evaluated in the preoperative assessment and dealt with according to each patient's needs. Even though this article focuses on isolated management of the aging neck, careful evaluation of the neck and its relationship to the lower third of the face is fundamental. If there is significant jowling and descent of the neck-face interface, an isolated neck-lift procedure will not address that problem and will lead to a suboptimal result. In these patients, a face and neck lift is a more appropriate operation.

KEYWORDS: Neck lift, cervicomental angle, platysma, SMAS

Neck lifting is the process of rejuvenation and contouring of the neck. The contour of the neck and the cervicomental angle are very important determinants of overall facial aesthetics (Fig. 1). Almost all face-lift procedures, especially those involving the superficial muscular aponeurotic system (SMAS), will have some effect on the neck, particularly the jawline. For a small group of patients, this may be all that is needed, whereas others will need more extensive procedures to improve the neck contour. Neck lifts can also be performed as separate procedures without a concomitant face-lift.

There are three surgical planes involved in this procedure: the superficial plane (subcutaneous fat), the intermediate plane (platysma muscles and the fat between the two muscles), and the deep plane (subplatysmal fat, the anterior belly of the digastric muscles, and the submandibular glands). ${ }^{1}$ These three planes need to be evaluated and addressed according to each patient's needs. Patients' necks do not have the same morphology, nor do patients age at the same rate or in the same way.

\section{HISTORY}

There are many notable surgeons who have influenced the thinking and current approach to the rejuvenation of the neck. Courtiss demonstrated that removal of submental fat with liposuction resulted in redraping and recontouring of the submental skin without any redundancy. ${ }^{1}$ Normal skin quality and elasticity are prerequisites. Feldman's corset platysmaplasty improved the submental contour and jawline and eliminated platysma bands. ${ }^{2}$ Connell's subplatysmal approach through medial platysmal elevation further improved neck contour through the elimination of bulging deep fat, digastric muscles, and submandibular glands. ${ }^{3}$

\section{ANATOMY}

Procedures in the neck are designed to modify the submental/submandibular region and the cervicomental angle by reduction or relocation of volume. The neck can be divided into three separate planes where the volume

\footnotetext{
${ }^{1}$ Private Practice, Medellin, Colombia; ${ }^{2}$ Paces Plastic Surgery, Atlanta, Georgia; ${ }^{3}$ Division of Plastic and Reconstructive Surgery, Clinical Care Center, Baylor College of Medicine, Houston, Texas.

Address for correspondence and reprint requests: Farzad R. Nahai, M.D., Paces Plastic Surgery, 3200 Downwood Circle, NW, Suite 640,

Contemporary Face-lift Techniques; Guest Editor, Larry H. Hollier, Jr., M.D., F.A.C.S.

Semin Plast Surg 2009;23:264-273. Copyright (C) 2009 by Thieme Medical Publishers, Inc., 333 Seventh Avenue, New York, NY 10001, USA. Tel: +1(212) 584-4662.

DOI 10.1055/s-0029-1242178. ISSN 1535-2188. 

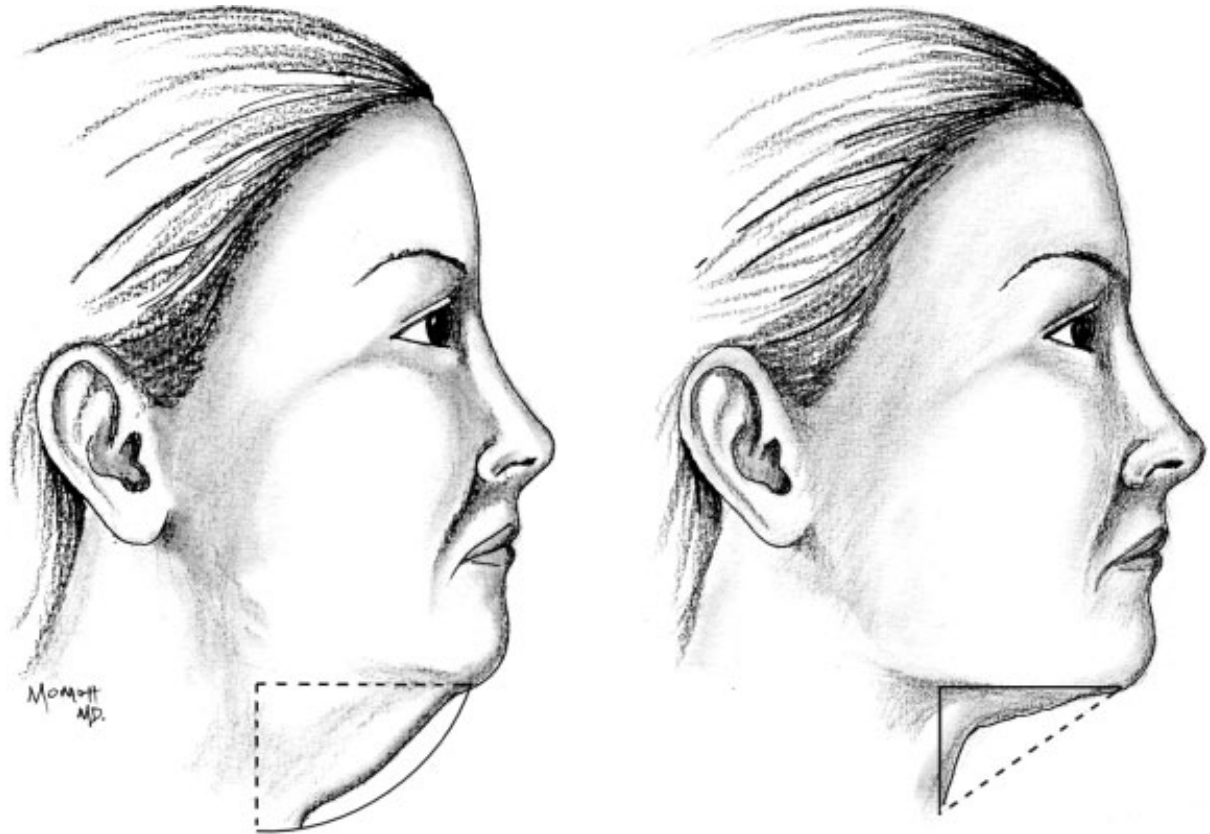

Figure 1 (Left) Lateral view of a patient with an obtuse cervicomental angle. (Right) Patient with a desirable cervicomental angle.

exists: the superficial plane, the intermediate plane, and the deep plane. ${ }^{4}$

\section{The Superficial Plane}

The superficial plane includes only the subcutaneous fat. The subcutaneous fat is found between the skin and the platysma muscle (Fig. 2). The thickness of this fat pad will vary according to the patient's morphology and weight. It is usually more abundant in the submental area, extending in between the platysma muscles if they are separated at this level.

\section{The Intermediate Plane}

The intermediate plane includes the platysma and any fat lying between the two muscles. The platysma separates the neck into superficial and deep compartments. These are large, thin, paired muscles that extend from the SMAS in the face, across the neck, and down to the clavicles. In the submental region, the anatomy of the platysma varies from individual to individual. Cardoso de Castrós anatomic studies led him to classify the relationship of the two platysma muscles and their interdigitation or decussation in the submental area into three types (Fig. 3):

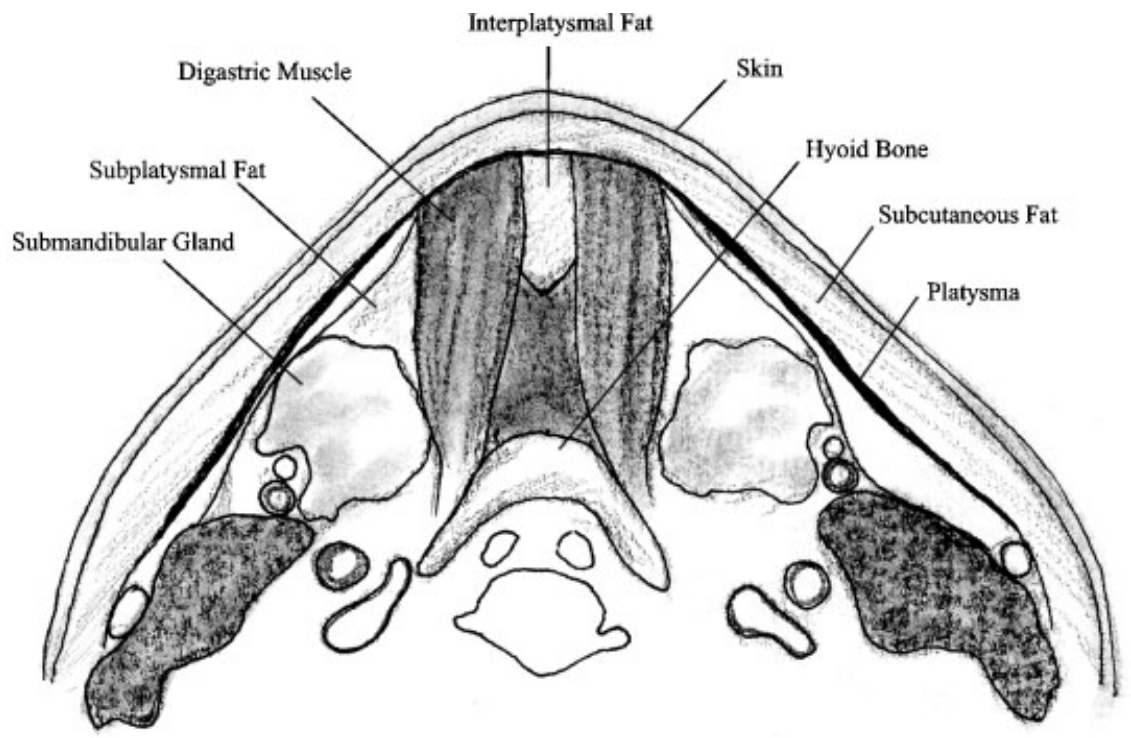

Figure 2 Surgical planes of the neck. 


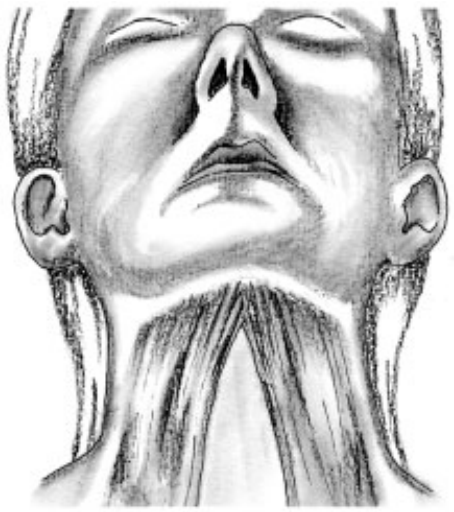

TYPE I

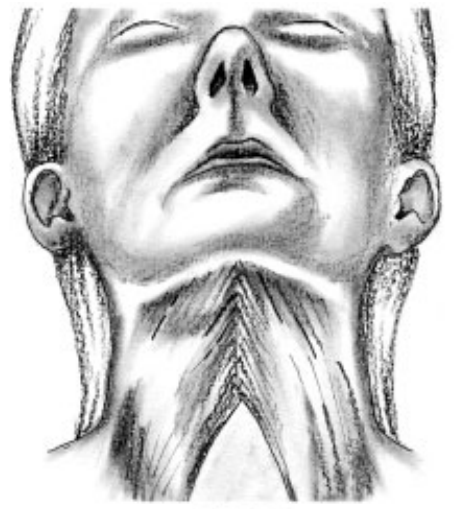

TYPE II

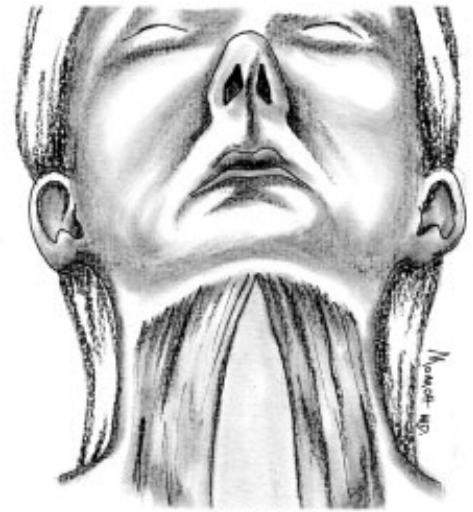

TYPE III

Figure 3 Cardoso de Castro's anatomic classification of the platysma muscles.

Type I: This is the most common anatomic variation. It is present in $75 \%$ of the population. Here, there is a limited decussation of the platysma muscles, extending 1 to $2 \mathrm{~cm}$ below the mandibular symphysis.

Type II: This variation is present in $15 \%$ of the population. These patients present a decussation of the platysma from the mandibular symphysis to the thyroid cartilage.

Type III: This is the least common anatomic variation. It is present in $10 \%$ of the cases. In these patients, there is no decussation of the platysma muscles at the midline.

Ascribing to this classification, the majority of patients have very limited decussation of the muscles medially. With age, the retaining ligaments that maintain the free medial edges approximated to the deep cervical fascia attenuate. Attenuation of this retaining ligament system allows these medial edges to descend resulting in the formation of the platysma bands. $\mathrm{Pa}^{-}$ tients with minimal or no platysmal decussation can have interplatysmal fat in the submental region between the medial edges of the two muscles. This fat is considered a structure of the intermediate plane but is contiguous with the subplatysmal fat of the deep plane.

\section{The Deep Plane}

The deep plane includes the subplatysmal fat, the digastric muscles, and the submandibular glands (Fig. 4). The

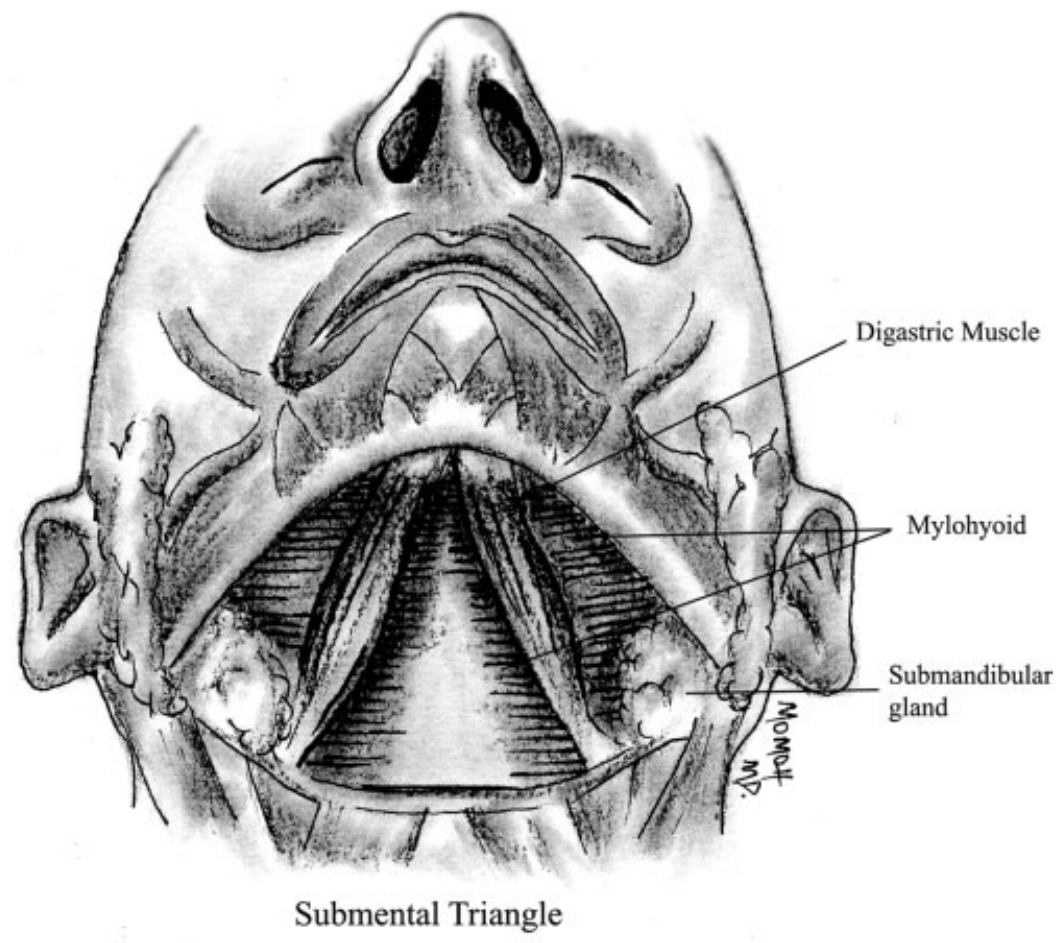

Figure 4 Submental triangles. 
subplatysmal fat is deep to the platysma muscles and superficial to the digastric muscles and the submandibular glands. The digastric muscles are paired bilateral structures that have an anterior and a posterior belly. The anterior bellies extend between the posterior surface of the mandible below the symphysis to the lesser cornu on each side of the hyoid bone. The anterior belly and posterior belly of each digastric muscle form two sides of the submental triangle. The third side of the triangle is the inferior edge of the body of the mandible. Within each triangle lie the submandibular gland, the facial artery and vein, the lingual nerve, and the marginal mandibular branch of the facial nerve. Motor innervation of the anterior belly of the digastric muscle is through a branch of the mandibular division of the trigeminal nerve. These muscles function as weak depressors of the mandible, and their excision proceeds without noticeable alteration in function.

The submandibular glands (salivary glands) are located in the submental triangles. When ptotic or hypertrophied, these structures can produce a visible bulge that disrupts the planar, smooth surface of a youthful-appearing neck. The facial artery and vein run over the posterior portion of the gland. The marginal mandibular branch of the facial nerve crosses the gland superficially. ${ }^{3}$ Both the lingual nerve and the hypoglossal nerve lie deep to the gland. These vascular and neural structures are located in an extracapsular location, thus the safest approach to resection of the gland is intracapsular, as these structures lie outside of the capsule of the gland.

\section{PREOPERATIVE ASSESSMENT}

Careful evaluation of the neck and its relationship to the lower third of the face will lead to selection of the appropriate procedure to meet the patient's goals. In order of importance in neck recontouring and rejuvenation, all three planes from superficial to deep are assessed; the subcutaneous fat, the platysma muscles, the digastric muscles, and the submandibular glands. Finally, the skin is evaluated for excess and elasticity. The interface of the neck and face (jowls and jawline) is also assessed (Table 1).

\section{Fat}

Fat removal has the most dramatic effect on neck recontouring. Therefore, it is important to determine the accurate location of fat in the neck, whether it is only under the skin or also between and below the platysma muscles. To differentiate between subcutaneous fat and subplatysmal bulk (deep fat and anterior belly of the digastric muscles), the surgeon pinches the submental area with the patient at rest and then after contraction of the platysma muscles (Fig. 5). If the amount of fat within

\section{Table 1 Focus of Neck Evaluation}

- Fat

- Muscles

- Submandibular gland

- Skin

- Neck-face interface

the pinch diminishes on contraction of the muscle, then a significant amount of bulk lies deep to the muscle, indicating that subcutaneous fat removal alone (liposuction) will not result in an ideal contour. In these cases, deep plane procedures would be necessary to reach our goal.

\section{Platysma Muscles}

The platysma bands may be visible at rest or on animation only. The patient is instructed to flex her platysma muscles to accentuate the visibility of the bands. The location of the bands and the distance between them is noted. Thyroid cartilage position and prominence are
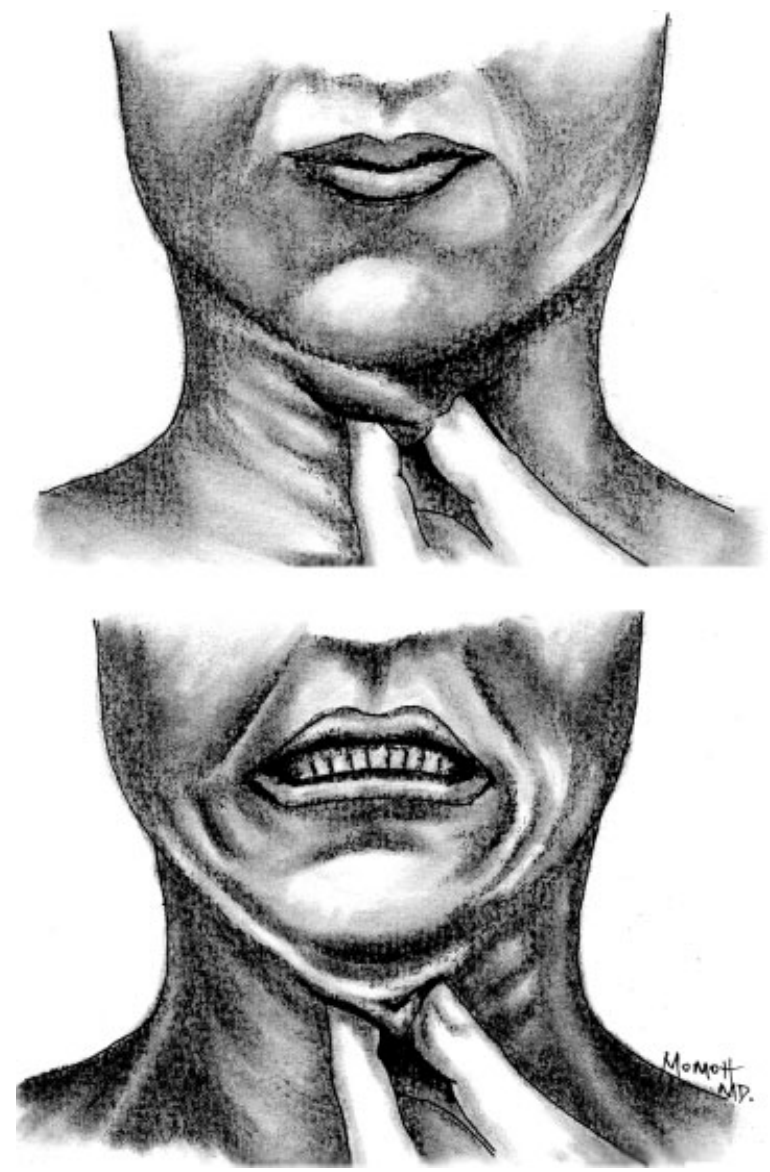

Figure 5 (Left) Evaluation of the location of the submental neck fat by pinching the submental area at rest. (Right) Subcutaneous versus subplatysmal fat is assessed by pinching the submental area during contraction. 
noted in female patients in whom a platysmal myotomy is being considered. The myotomy is planned low enough ( $2 \mathrm{~cm}$ below the cartilage) so that thyroid cartilage prominence is not unmasked, thus producing a masculine appearance.

\section{Digastric Muscles}

With the head in neutral position, the bulk in the region of the anterior belly of the digastric muscles is visualized and palpated. If flexion of the neck results in a bulge, it is indicative of subplatysmal bulk, and digastric muscle excision should be considered. However, transcutaneous evaluation of the digastrics may not always be possible, especially in a heavy neck, and therefore they are best evaluated during the procedure after the subcutaneous fat is removed.

\section{Submandibular Gland}

The submandibular gland is often visible transcutaneously, presenting as a bulge within the submental triangle. It is inspected by palpation of a discrete firm mass that can often be balloted against the medial and/or caudal surface of the body of the mandible. In a heavy neck, it might not be visible or palpable, and intraoperative evaluation may be necessary before making the surgical decision to excise it.

\section{Skin}

The length of the skin incision in neck lifting reflects the quality and quantity of skin that is present. A neck with good-quality skin and little or no excess can be successfully managed through a short scar submental approach. Patients who have excess, inelastic, sun-damaged skin must have the full retroauricular incision for a good result. It is important to evaluate whether the skin excess is real or apparent, and if real, where that skin excess lies. Apparent excess skin has adequate elasticity and will redrape following the contouring below the skin (Fig. 6). Real excess skin usually extends below the thyroid cartilage and posteriorly beyond the sternocleidomastoid muscle.

Skin evaluation also influences the choice of vectors. In combination with a face-lift, the vertical vector applied to the face, through SMAS and skin elevation, will define and improve the jawline. The more diagonal-posterior vector required for skin resection in the retroauricular area will define and improve the lower and lateral neck.

\section{Neck-Face Interface}

If there is significant jowling and descent of the neckface interface, an isolated neck-lift procedure will not address that problem and will lead to a suboptimal result. In these patients, a lower face-lift and neck lift is a more appropriate operation.

\section{PREOPERATIVE PLANNING}

Through the clinical evaluation of the neck, the planes of intervention are determined. If only subcutaneous fat excision is needed, liposuction in the superficial plane would be the procedure of choice. If platysma bands have to be dealt with, an intermediate plane intervention is planned. If subplatysmal fat removal, modification of the digastric muscles, and/or submandibular gland resection is required, intervention in the deep plane is performed. If the patient presents with jowls and aging of the neckface interface, concomitant face-lift procedures should be used. It is not rare to perform procedures in all three planes.

Four options for neck recontouring or rejuvenation, ranging from liposuction to a full-scar face and neck lift, are presented:

1. Liposuction

2. Submental neck lift

3. Short-scar face and neck lift

4. Full-scar face and neck lift

\section{OPERATIVE TECHNIQUE}

\section{Liposuction}

The best candidates for liposuction recontouring of the neck are usually younger patients with normal-quality skin and localized excess submental fat. Clinical evaluation of the platysma muscles and subplatysmal fat is key to patient selection. In addition to the pinch test described earlier, the patient's neck is examined at rest and on animation for the presence of platysma bands. If platysma bands are present, the patient would be best served by a submental lift. Even if the bands are only visible on animation, subcutaneous fat removal will expose them.

Liposuction of the neck may be performed under local or general anesthesia. The patient is placed supine with the neck in full extension. Through an access incision in the submental area and optional incisions behind each earlobe, the neck is infiltrated with a wetting solution. Ringer's lactate solution is used with $250 \mathrm{mg}$ lidocaine and $1 \mathrm{mg}$ epinephrine added to each liter. For the neck, $100 \mathrm{~mL}$ of this solution is more than adequate for ultrasound assisted lipoplasty (UAL) and suction-assisted liposuction (SAL). For ultrasonic liposuction of the neck, a short 2- to 3-mm solid probe is used and set at 50\% energy with the Mentor Contour Genesis UAL device (Mentor Corp., Santa Barbara, CA). The UAL phase is usually no longer than 2 to 

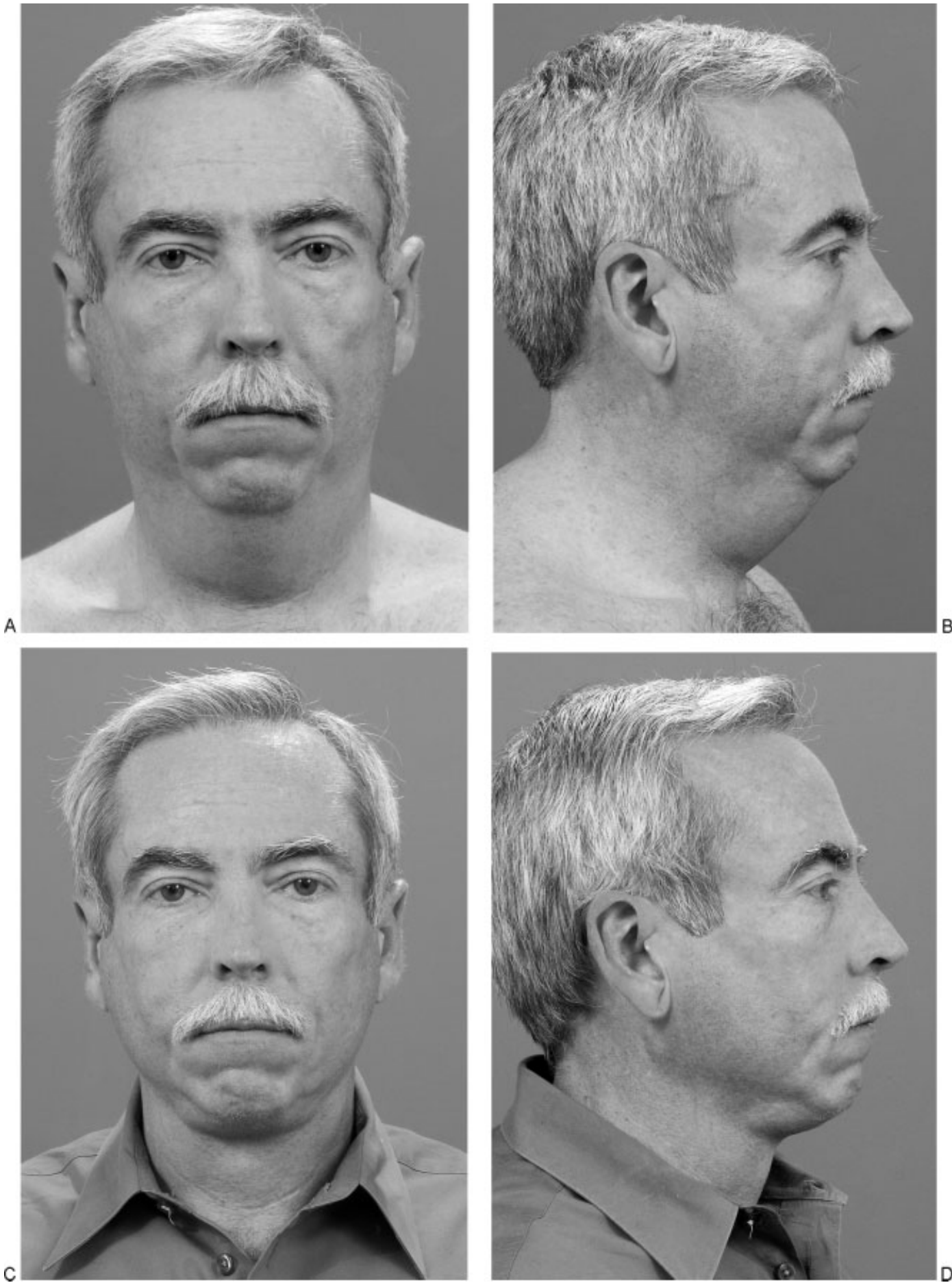

Figure 6 (A, B) Preoperative photos of 49-year-old patient. (C, D) Postoperative photos 9 months after neck lift. Skin redraping is demonstrated after superficial fat liposuction and platysma plication through a submental incision. No neck skin was excised.

3 minutes. This is followed by aspiration with a flat 2- to 3-mm cannula. For SAL of the neck, a flat 2- to 3-mm single-hole cannula is used. The suction is started through the submental incision with the hole always toward the deep tissue and never toward the skin. The cannula is passed slowly fanning out from the incision. Repeated passes in one tunnel will lead to oversuction and irregularities. If suction of the jawline and lateral neck is also indicated, the separate incision behind each earlobe is used as further access. It is important to leave at least $5 \mathrm{~mm}$ of fat on the deep surface of the skin to avoid postoperative irregularities. Oversuctioning under the dermis will lead to adherence of the skin to the underlying platysma, resulting in tethering and banding. 


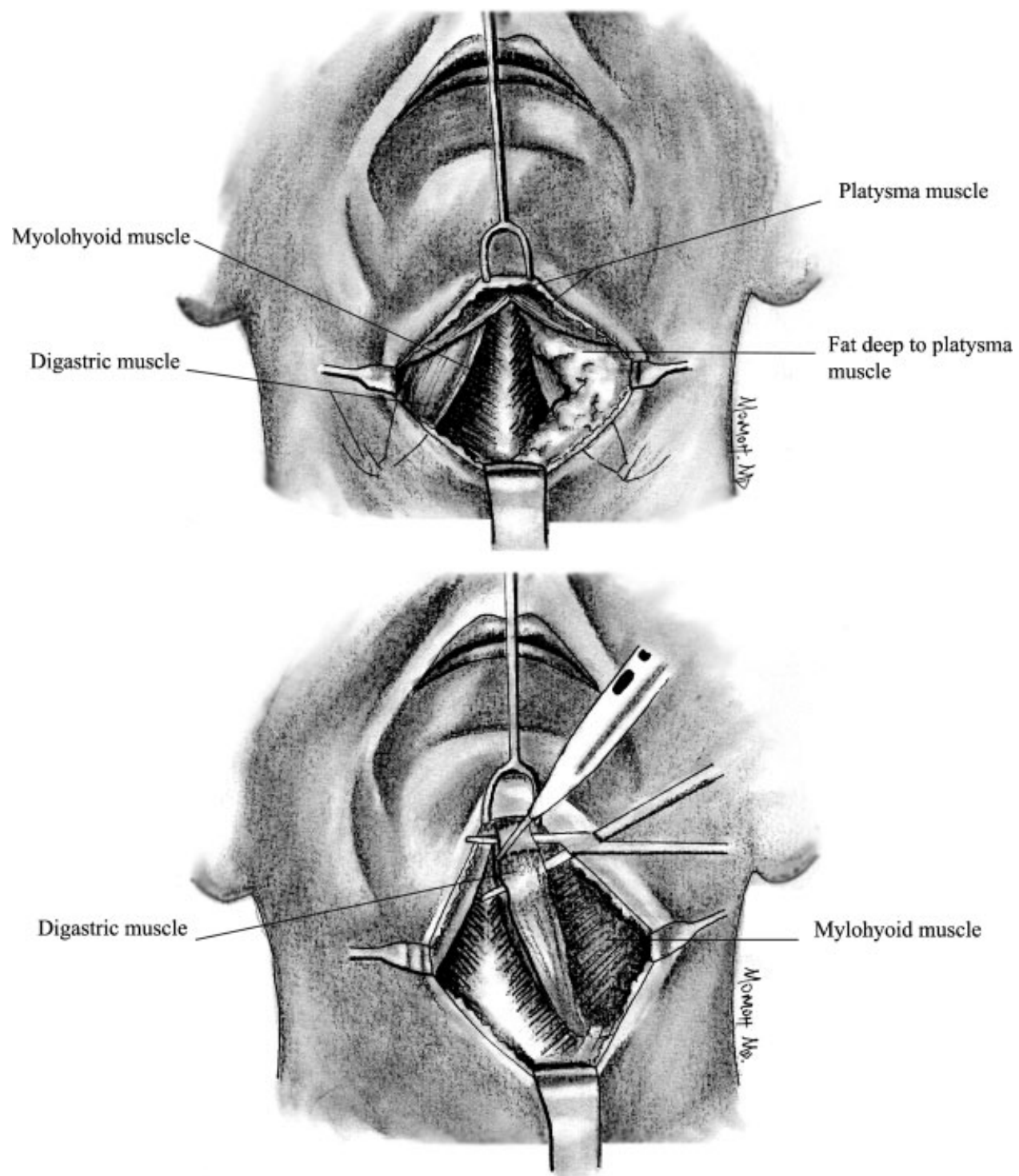

Figure 7 (Above) Elevation of the platysma muscles to expose the deep fat, the digastric muscles, and the submandibular glands. (Below) Tangential excision of the anterior belly of the digastric muscle preserving half of the thickness of the muscle.

No drains are required, but postoperative compression is used either with foam tape or an elastic garment.

\section{Submental Neck Lift}

The submental neck lift allows intervention in the intermediate (platysmal) plane and in the deep (subplatysmal) plane. The majority of intermediate and deep plane procedures are preceded by liposuction of the superficial plane. The patient is placed supine with the neck extended. If the area has not been infiltrated, a dilute lidocaine and epinephrine solution is used (usually $0.5 \%$ Xylocaine with epinephrine 1:200,000). An incision 3 to $5 \mathrm{~cm}$ in length is made parallel to and just posterior to the submental crease. The submental flap is raised leaving at least $5 \mathrm{~mm}$ of fat deep to the skin. The dissection is carried forward and laterally releasing the mandibular ligaments. The extent of the dissection is individualized. In most patients, the dissection is carried to at least the thyroid cartilage. After the skin flap is elevated, direct subcutaneous defatting is performed from the deep surface of the skin making sure to leave sufficient subcutaneous tissue $(5 \mathrm{~mm})$ of even thickness. The fat remaining on the platysma is suctioned or excised, and the fat between the platysma muscles is removed with direct scissors or bovie.

Access to the subplatysmal deep plane is obtained in a medial to lateral approach. In patients with type III platysmal anatomy, the medial edges of the platysma are elevated with scissor spreading dissection. In patients with platysma decussation (types I and II), a midline incision is made in the platysma with the coagulating current to create two free medial edges. The platysma is elevated to the level of the thyroid cartilage and laterally 
far enough to expose the subplatysmal fat, the anterior bellies of the digastric muscles, and the submandibular glands (Fig. 7).

Subplatysmal fat is managed by direct excision in a conservative manner. If the anterior bellies of the digastric muscles are to be left unmodified, the interplatysmal and subplatysmal fat is resected only to the caudal surface of the muscles to prevent the formation of a submental depression. If a muscle reduction is planned, fat is resected to the projected level of the muscle excision.

Once the anterior bellies of the digastric muscles can be easily visualized, they can be partially excised, totally excised, or the two plicated in the midline. A tangential excision is most commonly performed. This starts anteriorly at the origin of the muscle. A hemostat is passed halfway through the thickness of the muscle to separate the fibers that will be excised from those that will be left in place. The tips are spread, and with electrocautery the muscle fibers are transversely divided anteriorly and posteriorly so that half the thickness of the muscle is tangentially excised (Fig. 7). When a total excision is performed, the anterior origin of the digastric is cut first with electrocautery and then the tendinous junction is divided.

Once the anterior belly of the digastric muscle has been dealt with, an enlarged submandibular gland is easily visible. Before resection, the glands are reassessed by direct inspection to reconfirm the surgical plan. The gland is brought into view with a suture or Allice clamp. The most important step in resecting the gland is to incise the capsule and to perform the resection piecemeal. This will avoid damage to all the extracapsular vessels and nerves that are near the gland. Therefore, after entry into the intracapsular space, electrocautery is used to resect the gland within the capsule in an incremental fashion. The neck is flexed and re-evaluated with the skin redraped periodically to prevent overresection until the desired contour is seen.

After management of the structures responsible for deep plane bulk (deep fat, anterior bellies of the digastric muscles, and submandibular glands), attention is paid to the platysma muscles. The goal of platysmal management is to restore support to this structure and improve the cervicomental angle. For an isolated submental neck lift, the platysma muscles are plicated in the midline. The repair is usually performed in two layers from the mentum to the inferior edge of the thyroid cartilage. Interrupted sutures with permanent material are used for the first layer and an absorbable suture for the second layer. If work on the deep plane has been done, a small drain is left below the platysmal muscles. After completion of the midline plication, horizontal myotomy of the medial edges of the platysma is performed for a distance of $3 \mathrm{~cm}$ bilaterally at the caudal extent of the plication. The purpose of this is twofold. First, the myotomy allows the platysma to shift cepha- lically under the tension created by the medial repair and thus deepens the cervicomental angle. Second, the transaction helps to reduce the recurrence of platysma bands. For an isolated submental lift, the procedure at this stage is almost complete. Neck undermining extends laterally on either side until effective skin draping is seen. The skin incision is closed by planes, and finally tapes and dressings are applied.

\section{Short Scar Face and Neck Lift}

For patients who present with jowls and aging of the neck-face interface but no excess neck skin, a short scar face and neck lift is the procedure of choice. Neck recontouring through liposuction or through a submental approach may be performed at the same time. In this procedure, the diagonal upward vector on the SMAS and upward vector on the facial skin will improve the jawline significantly. The facial portion of this procedure is performed through a prehairline incision below the sideburn and a preauricular incision down and around the earlobe. The facial flap is elevated and the dissection continued down into the neck, the dissection usually extends to the posterior border of the sternocleidomastoid. If a submental approach is made, the medial and lateral dissections are connected in the subcutaneous plane. A diagonal resection or plication of the SMAS is made extending from the zygomatic prominence toward the angle of the mandible laterally. The plication or resection continues along the lateral border of the platysma. A long-lasting suture is used on the SMAS and the lateral platysma. When plicating the lateral platysma, the vector and location of the traction force applied is more important than the method employed to generate the force. Therefore, the force should be applied inferior to the gonial angle and oriented in a vector perpendicular to the midline of the neck. The greatest force is applied in the location where the apex of the cervicomental angle is desired, the level of the hyoid bone (Fig. 8). After the lateral platysmaplasty, the facial skin is pulled directly upward with a slight diagonal vector posteriorly. The excess skin is resected at the sideburn and at the preauricular incisions and a twolayered skin closure is performed with a 4-0 long lasting absorbable suture to the dermis and a 6-0 rapidly absorbing catgut to the skin.

\section{Full Scar Face and Neck Lift}

The best candidates for a full scar face and neck lift are individuals with aging changes of the face and neck with inelastic and excess lower and posterior neck skin. The procedure is similar to the operation described previously, with the exception that the skin incision is continued around the earlobe posteriorly up to the level of the tragus or higher and then across into the occipital 


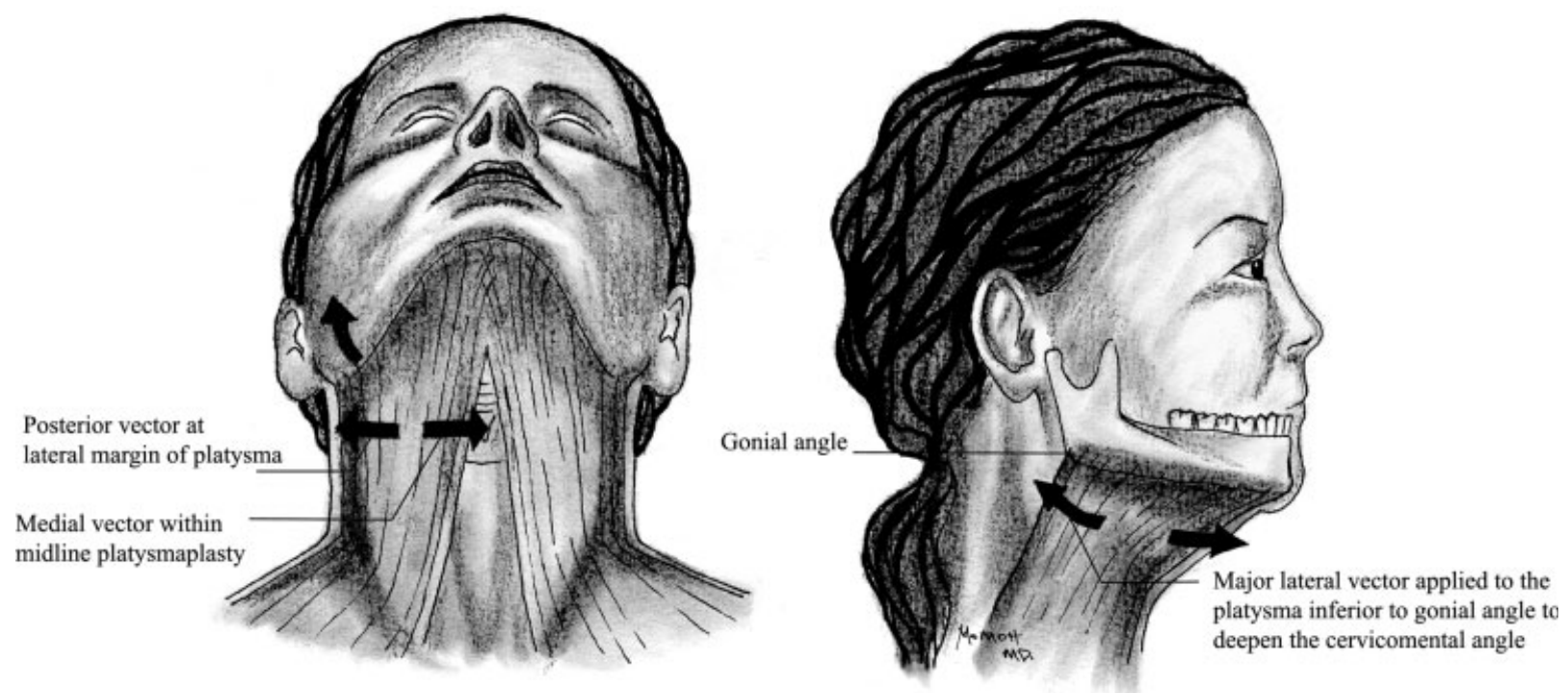

Figure 8 Vectors of platysmal tightening. Arrows demonstrate both vertical and horizontal vectors.

hair. The incision follows the occipital hairline, and its length is directly proportional to how much neck skin needs to be resected (Fig. 9). This incision will allow for ample resection of excess skin without altering the occipital hairline. There is more extensive skin undermining with this incision, extending beyond the posterior border of the sternocleidomastoid and behind the ear. Management of the SMAS and platysma was described previously. After lateral platysmaplasty, the facial skin is pulled directly upward with a slight diagonal vector posteriorly, and the retroauricular skin flap is pulled in a posterior diagonal vector assessing the best direction to obtain the ideal cervicomental contour. After the redraping and skin trimming, the incision in the face is closed as previously described. The excess neck skin is trimmed at the level of the retroauricular incision. When closing behind the ear, it is important to include the deep fascia in the retroauricular sulcus within the closure; this will prevent outward migration of the retroauricular scar. A drain is placed subcutaneously for the face and neck dissection.

\section{POSTOPERATIVE CARE}

Drains are placed in all surgical neck-lift procedures. Right after the procedure, a postoperative dressing is applied making sure it is not too tight. Most patients undergoing an isolated neck lift are allowed to go home the same day as surgery. Patients with neck lifts associated with face-lifts usually stay the night for observation. The following morning, the operative dressing and the drains are removed. After this, a compressive, nonbinding garment is worn for 5 days continuously, and then for 10 days at night only. Patients should be

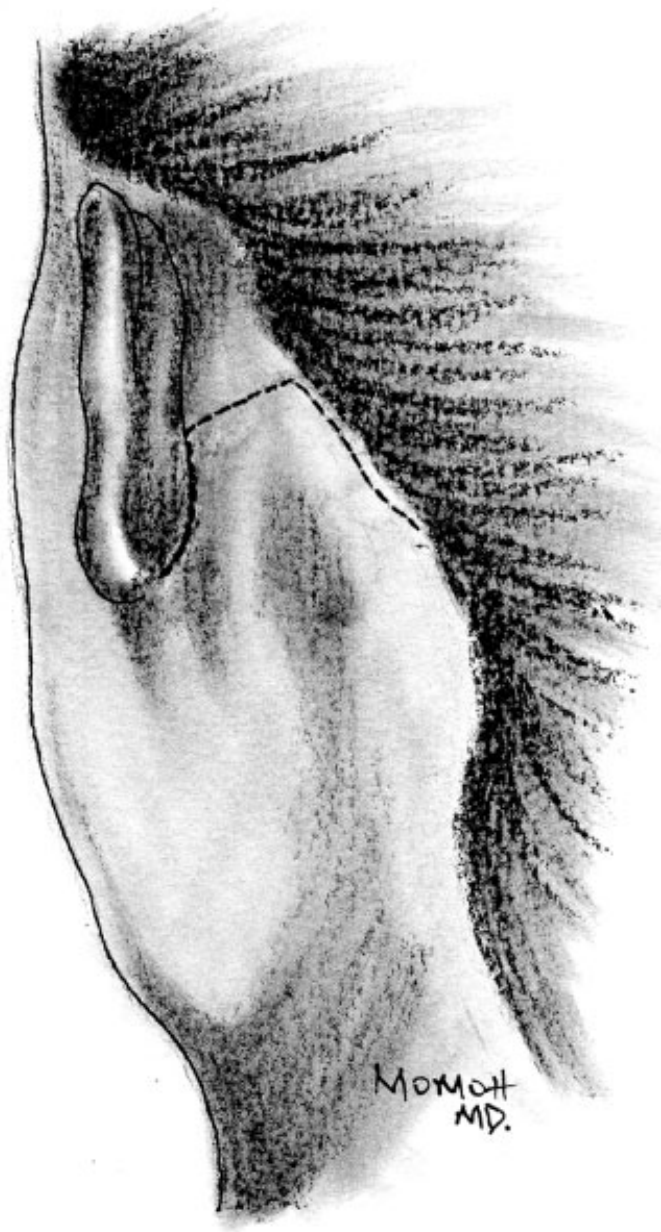

Figure 9 An occipital hairline incision is best suited for individuals with excessive or massive skin redundancy, especially in the lower neck. 
instructed to position their heads so that their necks are gently extended. Cervical flexion can increase edema through venous obstruction.

\section{COMPLICATIONS}

The most frequent complications in neck lifting are aesthetic in nature. The most common complication is contour irregularities due to overzealous superficial liposuction. This excessive removal of subcutaneous fat leads to adherence of the denuded dermis directly onto the platysma muscle leading to irregularities. The tendency to overdo the removal of superficial fat is related to a reluctance to deal with the deeper layers of the neck. In superficial liposuction, small cannulas turning the hole away from the skin should be used, and at least $5 \mathrm{~mm}$ of fat should always be left under the skin. It is always better to do less than more. The correction of untreated platysmal and subplatysmal planes is straightforward, but improvement of skin-platysmal adhesions is very difficult. For these problems, full mobilization of the skin off the platysma with secondary redraping offers some improvement. Autologous fat or dermis grafts are also used.

Another common aesthetic error in neck lift is related to vectors. In some patients, excessive posterior traction on the neck without adequate vectors of elevation on the face along the jawline will lead to a banding or a "hammock effect." In these cases, the lower and posterior neck are effectively tightened, but laxity remains in the submental area, jowls, and neckline.

The most frequent encountered problem in the platysma is the persistence of platysma bands. These occur when the bands are not dealt with primarily or when the plication sutures fail. Reoperation or the temporary treatment with botulinum toxin is the solution. A persistent submental bulge after neck contouring is related to the hypertrophic digastric muscles or submandibular glands, which may have been unmasked when subcutaneous fat was removed. Correction entails operation of the deep plane.

Acute complications include hematomas, seromas, and injury to motor and sensory facial nerves. The rate of hematomas in neck lifts is $3 \%$, and it is not increased with the addition of deep plane procedures. ${ }^{5}$ Expanding hematomas represent a surgical emergency due to the potential for airway compromise and skin flap necrosis. If a seroma forms, aspiration at regular intervals is performed until it resolves. If the patient had subtotal submandibular gland resections with fluid collection appearing soon after drain removal and the patient reports enlargement with meals, a sialoma may be present. In these cases, consideration should be given to aseptically replacing a drain and/or the initiation of a systemic glycopyrolate administration to reduce saliva production.

The great auricular nerve (sensory nerve form the cervical plexus) is the most commonly injured nerve during full scar face and neck lifts. It is at greatest risk during the retroauricular dissection in its superficial location on the sternocleidomastoid muscle $6.5 \mathrm{~cm}$ below the external auditory canal. Division of the nerve will lead to numbness of portions of the ear. Injury to the marginal mandibular branch of the facial nerve results in weakness of depression of the ipsilateral hemi-lower lip due to denervation of the depressor anguli oris and mentalis muscles. In most cases, it is traction injury that is not permanent, and function returns in 24 hours to 3 months.

\section{CONCLUSION}

A very pragmatic approach to neck lifting is presented. Dividing the neck in three surgical and anatomic planes allows us to perform a thorough evaluation of the patient's needs and will guarantee the patient a better postoperative result. Overoperating in the superficial plane to avoid deeper surgery will only unmask the problem and make for an unsatisfied patient. The quality and quantity of the neck skin is crucial when choosing an incision. Lastly, remember that the neck should be in harmony with the lower face, and if there is significant jowling and descent of the neck-face interface, an isolated neck-lift procedure will not address that problem and will lead to a suboptimal result. In these patients, a lower face and neck lift with a short or full scar is a more appropriate operation.

\section{REFERENCES}

1. Nahai F. Neck lift. In: Nahai F, ed. The Art of Aesthetic Surgery. St. Louis, MO: Quality Medical Publishing; 2005: 1239-1283

2. Connell BF, Shamoun JM. The significance of digastric muscle contouring for rejuvenation of the submental area of the face. Plast Reconstr Surg 1997;99:1586-1590

3. Ellenbogen R, Karlin JV. Visual criteria for success in restoring the youthful neck. Plast Reconstr Surg 1980;66:826-837

4. Nahai F, Nahai FR, Ford DT. Applied anatomy of the face and neck. In: Nahai F, ed. The Art of Aesthetic Surgery. St. Louis, MO: Quality Medical Publishing; 2005:827-896

5. Nahai F, Bryce A. Deep plane procedures in the neck. In: Aston SJ, Steinbrech D, Walden J, eds. Aesthetic Plastic Surgery. New York: Elsevier; 2009:231-242

\section{SUGGESTED READINGS}

Feldman JJ. Corset platysmaplasty. Plast Reconstr Surg 1990;85: 333-343

Fuente del Campo A. Midline platysma muscular overlap for neck restoration. Plast Reconstr Surg 1998;102:1710-1714; discussion 1715

Guerrerosantos J. Neck lift. Simplified surgical technique, refinements, and clinical classification. Clin Plast Surg 1983;10:379404

Nahai F. Reconsidering neck suspension sutures. Aesthet Surg J 2004;24:365-367 